\title{
Detection of Aflatoxin M1 Using a Dual Immunosensing Platform ${ }^{+}$
}

\author{
Ana-Maria Gurban 1,* , Petru Epure 2, Iuliana Raut ${ }^{1}$, Mariana Calin ${ }^{1}$, Gelu Vasilescu 1 , \\ Luiza Jecu ${ }^{1}$, Melania-Liliana Arsene ${ }^{1}$ and Mihaela Doni ${ }^{1, *}$ \\ 1 National Research \& Development Institute for Chemistry \& Petrochemistry-ICECHIM; Biotechnology \\ Department, 202 Spl. Independentei, 060021 Bucharest, Romania; iulia_rt@yahoo.com (I.R.); \\ marriconstantin@yahoo.com (M.C.); gelu_gvp@yahoo.com (G.V.); jecu.luiza@icechim.ro (L.J.); \\ melania_arsene@yahoo.com (M.-L.A.) \\ 2 EPI-SISTEM SRL, Bvd. Brasovului 145, 505600 Sacele, Brasov, Romania; petru.epure@epi.ro \\ * Correspondence: amgurban@yahoo.com (A.-M.G.); mihaela.doni@icechim.ro (M.D.) \\ + Presented at the 15th International Symposium "Priorities of Chemistry for a Sustainable Development" \\ PRIOCHEM, Bucharest, Romania, 30th October-1st November 2019.
}

Published: 9 October 2019

Keywords: mycotoxins; dual detection; immune-sensitive platform

Determination of aflatoxin M1 in milk and dairy products is of great interest, due to its toxicity and negative effects exerted over the humans and animal's health when fed products contaminated with mycotoxins. Aflatoxin M1 (AFM1) represents the major hydroxylated metabolite of aflatoxin B1 (AFB1), which can be detected in the tissue and biological fluids of animals consuming contaminated food with AFB1. This metabolite is excreted in the milk produced by the mammary glands of the infected subjects [1].

Aflatoxin M1 is found in very low concentrations in milk and milk products, due to its high stability, even during the milkprocessing stages (pasteurization, UHT, etc.). It may be present in dairy products at even higher concentrations than in raw milk. Immunosensors represent a suitable alternative that has grown in the last decades in the development of sensitive, selective, simple and reliable systems for mycotoxin detection. The use of specific monoclonal antibodies or aptamers as bioreceptors, coupled with a physical transducer such as gold, carbon or graphite, leads to miniaturization of the systems and to improvement of the sensitivity, speed and low cost of analysis [2].

A combined electrochemical and optical immunosensitive platform has been developed based on an electrosensitive material obtained by incorporation of a monoclonal antibody specific to AFM1, into a polymeric film of 2,6-dihyroxynaphthalene and 2-(4-aminophenyl)ethylamine electropolymerized onto the gold working electrode on a glass support. This allows sensitive detection of aflatoxin M1 from complex liquid samples, based on quasi-simultaneous detection, electrochemical and surface plasmon resonance (SPR).

The principle of AFM1 detection from liquid samples using the combined dual detection platform is based on surface direct competition for the binding sites of the specific anti-AFM1 antibody immobilized in the polymeric film, between bioconjugate AFM1-HRP and target analyte AFM1. The immuno-recognition process is monitored indirectly, by electrochemical detection of the enzyme activity of horseradish peroxidase (HRP) used as marker in bioconjugate and, directly, by optic detection using surface plasmon resonance (SPR).

The results showed a good accuracy for AFM1 determination, with a dynamic range of quantification between 10 and $400 \mathrm{ppt}$, and a good correlation between SPR and electrochemical determination of AFM1, which indicates a high analytical performance of the developed dual detection platform. 
Acknowledgments: The authors acknowledge to CCCDI-UEFISCDI, project number ERANET-MANUNET II -TOX-HAZ-ASSESS no 33/2017 and to Ministry of Research and Innovation, project PN 19.23.03.02/2019.

\section{References}

1. Milicevic, D.R.; Skrinjar, M.; Baltic, T. Real and Perceived Risks for Mycotoxin Contamination in Foods and Feeds: Challenges for Food Safety Control. Toxins 2010, 2, 572-592.

2. Goud, K.Y.; Sharma, A.; Hayat, A.; Catanante, G.; Gobi, K.V.; Gurban, A.-M.; Marty, J.-L. Tetramethyl-6-carboxyrhodamine quenching-based aptasensing platform for aflatoxin B1: Analytical performance comparison of two aptamers. Analyt. Biochem. 2016, 508, 19-24.

(C) 2019 by the authors. Licensee MDPI, Basel, Switzerland. This article is an open access article distributed under the terms and conditions of the Creative Commons Attribution (CC BY) license (http://creativecommons.org/licenses/by/4.0/). 